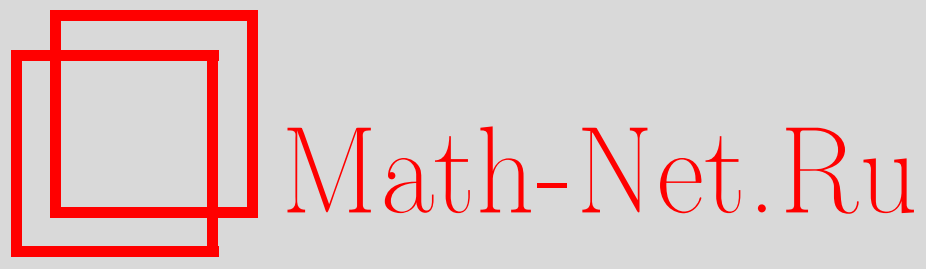

А. А. Клячин, В. М. Миклюков, Изотропные гиперповерхности и минимальные продолжения липшицевых функций, Функи. анализ и его прил., 2005, том 39, выпуск 3, 28-36

DOI: https://doi.org/10.4213/faa72

Использование Общероссийского математического портала Math-Net.Ru подразумевает, что вы прочитали и согласны с пользовательским соглашением

http://www . mathnet.ru/rus/agreement

Параметры загрузки:

IP : 52.205 .19 .152

26 апреля 2023 г., 18:26:27 
Функииональный анализ и его приложения

2005, т. 39, вып. 3, с. 28-36

УДК 517.95

\title{
Изотропные гиперповерхности и минимальные продолжения липшицевых функций
}

\author{
(c) 2005. А. А. Клячин, В. М. Миклюков
}

В работе устанавливается теорема существования и единственности для изотропных гиперповерхностей с заданным краем в искривленных лоренцевых произведениях. Доказательство базируется на минимальных липшицевых продолжениях функций.

1. Пусть $M$ - риманово многообразие класса $C^{2}$ с метрикой $g$, и пусть $\delta(m)>0$ есть $C^{2}$-функция на $M$. Пусть $H$ - пространство Лоренца с метрикой $h$. Следуя [1, разд. 2.6], искривленным лоренцевым произведением $\mathscr{W}=$ $M \times{ }_{\delta} H$ будем называть многообразие, оснащенное лоренцевой метрикой $\bar{g}$, определяемой по правилу

$$
\bar{g}(u, v)=g(\pi u, \pi v)+\delta(\pi(p)) h(\eta u, \eta v),
$$

где $p \in \mathscr{W}, \pi$ и $\eta$ - естественные проекции на $M$ и $H$ соответственно и $u, v \in$ $T_{p}(\mathscr{W})$. Из вида метрики ясно, что касательные пространства $T_{\pi(p)} M$ и $T_{\eta(p)} H$ ортогональны.

Вектор $u \in T_{p}(\mathscr{W})$ называется пространственноподобным, если $\bar{g}(u, u)>0$. В вырожденном случае, когда $\bar{g}(u, u)=0$, вектор $u$ называется изотропным (или световым). Будем говорить, что поверхность $F \subset \mathscr{W}$ является пространственноподобной, если всякий касательный к ней вектор является пространственноподобным. Поверхность $F$, в каждой точке которой найдутся как пространственноподобные, так и изотропные касательные векторы, будем называть изотропной (или световой).

Рассмотрим искривленное лоренцево произведение $\mathscr{W}$ вида $M \times_{\delta} \mathbb{R}$, где $\mathbb{R}-$ вещественная прямая с метрикой $-d t^{2}$. Далее будем рассматривать гиперповерхность $F$ в $\mathscr{W}=M \times{ }_{\delta} \mathbb{R}$, которая является графиком некоторой функции $f(m)$, непрерывно дифференцируемой в области $\Omega \subset M$.

Выпишем условия, при которых $F$ пространственноподобна. Фиксируем точку $m \in M$ и ортонормированный базис $\left\{E_{i}\right\}_{i=1}^{n}$ в $T_{m} M$. Пусть $E_{0} \in \mathbb{R}-$ некоторый вектор, для которого $\bar{g}\left(E_{0}, E_{0}\right)=-1$. При этом мы будем предполагать, что $E_{0}$ выбран таким образом, чтобы набор векторов $E_{0}, E_{1}, \ldots, E_{n}$ был ориентирован так же, как и $\mathscr{W}$.

Поверхность $F$ пространственноподобна тогда и только тогда, когда для всякого касательного векторного поля $X \in T F$ выполнено неравенство $\bar{g}(X, X)>0$. Представим $X$ в виде $X=\sum_{i=1}^{n} \xi_{i} X_{i}$, где $X_{i}=E_{i}+\nabla_{E_{i}}(f) E_{0}$ и $\nabla-$ связность в многообразии $M$. Тогда условие, что поверхность $F$ пространственно подобна, эквивалентно неравенству

$$
\sum_{i, j=1}^{n} \bar{g}\left(X_{i}, X_{j}\right) \xi_{i} \xi_{j}>0
$$


Ясно, что $X_{i} \in T F$. Действительно, зафиксируем точку $p=(m, t) \in F$. Пусть $\gamma(s) \subset M$ - произвольная гладкая кривая, удовлетворяющая условиям $\gamma(0)=m$, $\dot{\gamma}(0)=E_{i}$. Мы имеем $\gamma^{*}(s)=(\gamma(s), f(\gamma(s))) \subset F$ и $\gamma^{*}(0)=p$. Таким образом, для произвольной непрерывно дифференцируемой функции $h: F \rightarrow \mathbb{R}$

$$
\left.\frac{d}{d s} h(\gamma(s), f(\gamma(s)))\right|_{s=0}=\nabla_{E_{i}} h+\nabla_{E_{0}} h \nabla_{E_{i}} f=X_{i} h
$$

и, следовательно, вектор $X_{i}$ является касательным к поверхности $F$.

Соотношение (1) эквивалентно системе

$$
\operatorname{det}\left(\bar{g}\left(X_{i}, X_{j}\right)\right)_{i, j=1}^{k}>0, \quad k=1, \ldots, n .
$$

Вычислим определители. Мы имеем

$$
\operatorname{det}\left(\bar{g}\left(X_{i}, X_{j}\right)\right)_{i, j=1}^{k}=\operatorname{det}\left(\delta_{i j}-\delta(m) \nabla_{E_{i}} f \nabla_{E_{j}} f\right)=1-\delta(m) \sum_{i=1}^{k}\left(\nabla_{E_{i}} f\right)^{2} .
$$

Таким образом, график $F$ функции $t=f(m)$ пространственноподобен тогда и только тогда, когда

$$
\delta^{1 / 2}(m)|\nabla f(m)|<1 .
$$

Соответственно график $F$ является изотропным тогда и только тогда, когда

$$
\delta^{1 / 2}(m)|\nabla f(m)|=1 \quad \text { для всех } m \in \Omega .
$$

Предположим теперь, что многообразие $M$ связно, и пусть $\Omega$ - область в $M$. Рассмотрим внутреннюю метрику

$$
r_{\Omega}\left(m_{1}, m_{2}\right)=\inf \int_{\gamma} \delta^{-1 / 2}(m) d s,
$$

где $d s-$ элемент длины на $M$ и точная нижняя грань берется по всевозможным дугам $\gamma \subset \Omega$, соединяющим точки $m_{1}, m_{2} \in \Omega$. Пусть $\Omega_{r}-$ пополнение области $\Omega$ по метрике $r_{\Omega}$ и $\partial \Omega_{r}=\Omega_{r} \backslash \Omega$. Шар в метрике $r_{\Omega}$ с центром в точке $m$ и радиусом $R>0$ будем обозначать через $D_{R}(m)$, т. е.

$$
D_{R}(m)=\left\{m^{\prime} \in \Omega_{r}: r_{\Omega}\left(m, m^{\prime}\right)<R\right\} .
$$

Рассмотрим множество

$$
\Gamma\left(m_{1}, m_{2}\right)=\left\{m \in \Omega_{r}: r_{\Omega}\left(m_{1}, m_{2}\right)=r_{\Omega}\left(m_{1}, m\right)+r_{\Omega}\left(m, m_{2}\right)\right\} .
$$

Ясно, что $\Gamma\left(m_{1}, m_{2}\right) \neq \varnothing$, поскольку в этом множестве, во всяком случае, содержатся точки $m_{1}$ и $m_{2}$. Отметим также, что $\Gamma(m, m)=\{m\}$.

2. Предположим, что задана некоторая функция $\varphi: \partial \Omega_{r} \rightarrow \mathbb{R}$. В работах $[2,3]$ была исследована задача о пространственноподобном продолжении функции $\varphi$ с границы $\partial \Omega_{r}$ на всю область $\Omega$. Данная задача возникает, например, при описании множества допустимых функций в вариационной проблеме

$$
\int_{\Omega} \sqrt{1-\delta|\nabla f|^{2}} d v_{M} \rightarrow \max
$$

а также в вопросах разрешимости $[4,5]$ задачи Дирихле для уравнения

$$
\operatorname{div}\left(\frac{\delta \nabla f}{\sqrt{1-\delta|\nabla f|^{2}}}\right)=0
$$


В частности, доказано [3, Theorem 1], что функция $\varphi$ допускает пространственноподобное продолжение тогда и только тогда, когда

$$
\left|\varphi\left(m_{1}\right)-\varphi\left(m_{2}\right)\right| \leqslant r_{\Omega}\left(m_{1}, m_{2}\right) \text { для всех } m_{1}, m_{2} \in \partial \Omega_{r},
$$

причем

$$
\left|\varphi\left(m_{1}\right)-\varphi\left(m_{2}\right)\right|<r_{\Omega}\left(m_{1}, m_{2}\right),
$$

если $\Gamma\left(m_{1}, m_{2}\right) \backslash \partial \Omega_{r} \neq \varnothing$.

Возможность изотропного продолжения функции $\varphi$ на всю область $\Omega$ оставалась неисследованной. Ниже мы восполняем данный пробел. Опираясь на технику минимальных липшицевых продолжений, развиваемую в [6-9] в иных целях, мы указываем некоторый критерий изотропной продолжимости.

3. Пусть $X$ - метрическое пространство с метрикой $d$, и пусть $E-$ компактное подмножество в $X$. Предположим, что функция $\varphi: E \rightarrow \mathbb{R}$ определена на $E$ и удовлетворяет там условию Липшица

$$
\left|\varphi\left(x^{\prime}\right)-\varphi\left(x^{\prime \prime}\right)\right| \leqslant L d\left(x^{\prime}, x^{\prime \prime}\right) \quad \text { для любых } x^{\prime}, x^{\prime \prime} \in E .
$$

Наименьшую из постоянных $L$ в данном неравенстве будем обозначать через $\operatorname{Lip}(\varphi, E)$.

Всякая липшицева функция $\varphi: E \rightarrow \mathbb{R}$ может быть продолжена до липшицевой же функции $f: X \rightarrow \mathbb{R}$ так, чтобы $\left.f\right|_{E}=\varphi$ и $\operatorname{Lip}(\varphi, E)=\operatorname{Lip}(f, X)$ (см. $[10,2.10 .44])$. Функция $f$ называется в этом случае минимальным липиицевым продолжением функции $\varphi$. Подобные продолжения были предложены впервые, по-видимому, в $[13,14]$ в виде

$$
\begin{aligned}
& \bar{f}(x)=\inf _{y \in E}\{\varphi(y)+\operatorname{Lip}(\varphi, E) d(x, y)\}, \\
& \underline{f}(x)=\sup _{y \in E}\{\varphi(y)-\operatorname{Lip}(\varphi, E) d(x, y)\} .
\end{aligned}
$$

Нетрудно доказать, что если $\bar{f}$ и $\underline{f}$ суть указанные выше минимальные липшицевы продолжения функции $\varphi \frac{\bar{и}}{f}-$ произвольное минимальное липшицево продолжение той же функции, то

$$
\underline{f}(x) \leqslant f(x) \leqslant \bar{f}(x) \text { всюду в } X .
$$

Действительно, если $f(x)$ - минимальное липшицево продолжение, то для любых $x \in X, y \in E$

$$
|f(x)-\varphi(y)| \leqslant \operatorname{Lip}(\varphi, E) d(x, y) .
$$

Отсюда, например, следует, что

$$
f(x) \leqslant \varphi(y)+\operatorname{Lip}(\varphi, E) d(x, y),
$$

и, поскольку $y \in E-$ произвольная точка, получаем, что

$$
f(x) \leqslant \inf _{y \in E}\{\varphi(y)+\operatorname{Lip}(\varphi, E) d(x, y)\}=\bar{f}(x) .
$$

Аналогично доказывается неравенство $f(x) \geqslant \underline{f}(x)$. По поводу случая $X=\mathbb{R}^{n}$ cм. [6, Theorem 1].

Функции $\bar{f}$ и $f$ называются верхним и нижним продолжениями функции $\varphi$ соответственно. Следует заметить, однако, что минимальные липшицевы продолжения учитывают лишь глобальные липшицевы постоянные и в значительной мере игнорируют локальное строение функции. 
Множество

$$
U_{\varphi}=\{x: x \notin E, \bar{f}(x)=\underline{f}(x)\}
$$

называется множеством единственности функции $\varphi$.

Введем следующие обозначения:

$$
\begin{gathered}
\Gamma\left(x_{1}, x_{2}\right)=\left\{x \in X: d\left(x_{1}, x_{2}\right)=d\left(x_{1}, x\right)+d\left(x, x_{2}\right)\right\}, \\
I_{\varphi}=\bigcup\left\{\Gamma\left(x_{1}, x_{2}\right): x_{1}, x_{2} \in E \text { и }\left|\varphi\left(x_{1}\right)-\varphi\left(x_{2}\right)\right|=\operatorname{Lip}(\varphi, E) d\left(x_{1}, x_{2}\right)\right\} .
\end{gathered}
$$

На самом деле на множестве $X \backslash E$ множество единственности совпадает с множеством $I_{\varphi}$. А именно, справедливо следующее утверждение:

Теорема 1. Пусть $E \subset X$ - компактное множсество и $\varphi-$ заданная на нем липиицева функиия. Тогда

$$
U_{\varphi}=I_{\varphi} \backslash E
$$

ДокАзАтельство. Для определенности будем считать, что $\operatorname{Lip}(\varphi, E)=1$. Рассмотрим верхнее и нижнее продолжения $\bar{f}(x)$ и $\underline{f}(x)$ функции $\varphi$. Ясно, что $\bar{f}(x) \geqslant \underline{f}(x)$ при $x \in X$ и $\bar{f}(x)=\underline{f}(x)=\varphi(x)$ при $x \in E$.

Пусть $x_{0} \in I_{\varphi} \backslash E$. Покажем, что $\bar{f}\left(x_{0}\right)=f\left(x_{0}\right)$. Так как $x_{0} \in I_{\varphi}$, то существуют точки $y_{1}, y_{2} \in E$, для которых $\left|\varphi\left(y_{1}\right)-\bar{\varphi}\left(y_{2}\right)\right|=d\left(y_{1}, y_{2}\right)$ и $x_{0} \in \Gamma\left(y_{1}, y_{2}\right)$. Не ограничивая общности, будем предполагать, что

$$
\varphi\left(y_{1}\right)-\varphi\left(y_{2}\right)=d\left(y_{1}, y_{2}\right)
$$

По определению функции $\bar{f}$ имеем

$$
\bar{f}\left(x_{0}\right) \leqslant \varphi\left(y_{2}\right)+d\left(y_{2}, x_{0}\right) .
$$

Тогда, используя определение функции $f$, равенство (9), тот факт, что $x_{0} \in$ $\Gamma\left(y_{1}, y_{2}\right)$, и неравенство (10), получаем

$$
\begin{aligned}
\underline{f}\left(x_{0}\right) \geqslant \varphi\left(y_{1}\right)-d\left(y_{1}, x_{0}\right) & =\varphi\left(y_{2}\right)+d\left(y_{1}, y_{2}\right)-d\left(y_{1}, x_{0}\right) \\
& =\varphi\left(y_{2}\right)+d\left(y_{2}, x_{0}\right) \geqslant \bar{f}\left(x_{0}\right) .
\end{aligned}
$$

Следовательно, $\bar{f}\left(x_{0}\right)=\underline{f}\left(x_{0}\right)$.

Предположим теперь, что $x_{0} \in U_{\varphi}$. В силу компактности подмножества $E$ найдутся точки $y_{1}, y_{2} \in E$, для которых

$$
\bar{f}\left(x_{0}\right)=\varphi\left(y_{1}\right)+d\left(y_{1}, x_{0}\right), \quad \underline{f}\left(x_{0}\right)=\varphi\left(y_{2}\right)-d\left(y_{2}, x_{0}\right) .
$$

Taк как $x_{0} \in U_{\varphi}$, то

$$
d\left(y_{1}, y_{2}\right) \geqslant \varphi\left(y_{2}\right)-\varphi\left(y_{1}\right)=d\left(y_{1}, x_{0}\right)+d\left(y_{2}, x_{0}\right) .
$$

Следовательно, $x_{0} \in \Gamma\left(y_{1}, y_{2}\right)$ и $\varphi\left(y_{2}\right)-\varphi\left(y_{1}\right)=d\left(y_{1}, y_{2}\right)$, т. е. $x_{0} \in I_{\varphi}$.

4. Мы используем минимальные липшицевы продолжения для описания условий существования изотропной поверхности, натянутой на заданный контур. Прежде всего, несложно убедиться в том, что неравенство (5) эквивалентно условию

$$
I_{\varphi} \cap \Omega=\varnothing
$$

где множество $I_{\varphi}$ определено по отношению к метрике $r_{\Omega}$.

Нам понадобятся следующие два вспомогательных утверждения. 
Лемма 1. Пусть $M-$ риманово многообразие класса $C^{2}$ u $m_{0} \in M$. Положим

$$
r(m)=r_{\Omega}\left(m_{0}, m\right)
$$

Тогда в достаточно малой проколотой окрестности $D_{\varepsilon}\left(m_{0}\right) \backslash\left\{m_{0}\right\}$ функиия $r(m)$ непрерывно дифберенцируема.

Доказывается данное утверждение так же, как и в случае бесконечно гладкого многообразия (см., например, [12, разд. 8.1]).

Прежде чем перейти к следующему вспомогательному утверждению, отметим, что для любой подобласти $B$, компактно лежащей в $\Omega$, имеют место равенства

$$
\begin{aligned}
& \bar{f}(m)=\inf _{m^{\prime} \in \partial B}\left\{\bar{f}\left(m^{\prime}\right)+r_{\Omega}\left(m, m^{\prime}\right)\right\}, \\
& \underline{f}(m)=\sup _{m^{\prime} \in \partial B}\left\{\underline{f}\left(m^{\prime}\right)-r_{\Omega}\left(m, m^{\prime}\right)\right\} .
\end{aligned}
$$

Докажем, например, первое из них. Положим

$$
\bar{f}^{*}(m)=\inf _{m^{\prime} \in \partial B}\left\{\bar{f}\left(m^{\prime}\right)+r_{\Omega}\left(m, m^{\prime}\right)\right\} .
$$

Из неравенств $(7)$ следует, что $\bar{f}^{*}(m) \geqslant \bar{f}(m)$. Пусть $m \in \Omega$ и последовательность точек $m_{k} \in \partial \Omega_{r}$ такова, что

$$
\bar{f}(m)=\varphi\left(m_{k}\right)+r_{\Omega}\left(m, m_{k}\right)-\varepsilon_{k},
$$

где $\varepsilon_{k} \geqslant 0$ и $\varepsilon_{k} \rightarrow 0$ при $k \rightarrow \infty$. Тогда найдутся точки $m_{k}^{\prime} \in \partial B \cap \Gamma\left(m, m_{k}\right)$. Действительно, для произвольного $\varepsilon>0$ рассмотрим кривую $\gamma_{\varepsilon}:[0,1] \rightarrow \Omega_{r}$, удовлетворяющую условиям

$$
\gamma_{\varepsilon}(0)=m, \quad \gamma_{\varepsilon}(1)=m_{k}
$$

и

$$
\int_{0}^{1} \delta^{-1 / 2} g\left(\dot{\gamma}_{\varepsilon}(t), \dot{\gamma}_{\varepsilon}(t)\right) d t \leqslant r_{\Omega}\left(m, m_{k}\right)+\varepsilon
$$

Возьмем некоторые точки $m_{k}^{\varepsilon} \in \partial B \cap \gamma_{\varepsilon}$. В силу компактности границы $\partial B$, не ограничивая общности, можно считать, что найдутся точки $m_{k}^{\prime} \in \partial B$, такие, что $m_{k}^{\varepsilon} \rightarrow m_{k}^{\prime}$ при $\varepsilon \rightarrow 0$. Нетрудно видеть, что

$$
\begin{aligned}
r_{\Omega}\left(m, m_{k}^{\varepsilon}\right)+r_{\Omega}\left(m_{k}^{\varepsilon}, m_{k}\right) & \leqslant \int_{0}^{t_{k}^{\varepsilon}} \delta^{-1 / 2} g\left(\dot{\gamma}_{\varepsilon}(t), \dot{\gamma}_{\varepsilon}(t)\right) d t+\int_{t_{k}^{\varepsilon}}^{1} \delta^{-1 / 2} g\left(\dot{\gamma}_{\varepsilon}(t), \dot{\gamma}_{\varepsilon}(t)\right) d t \\
& =\int_{0}^{1} \delta^{-1 / 2} g\left(\dot{\gamma}_{\varepsilon}(t), \dot{\gamma}_{\varepsilon}(t)\right) d t \leqslant r_{\Omega}\left(m, m_{k}\right)+\varepsilon .
\end{aligned}
$$

Устремляя $\varepsilon$ к 0 в этом соотношении, получаем неравенство

$$
r_{\Omega}\left(m, m_{k}^{\prime}\right)+r_{\Omega}\left(m_{k}^{\prime}, m_{k}\right) \leqslant r_{\Omega}\left(m, m_{k}\right),
$$

откуда в силу неравенства треугольника вытекает, что $m_{k}^{\prime} \in \Gamma\left(m, m_{k}\right)$. Из доказанного следует, что

$$
\begin{aligned}
\bar{f}^{*}(m) \leqslant \bar{f}\left(m_{k}^{\prime}\right)+r_{\Omega}\left(m_{k}^{\prime}, m\right) & \leqslant \varphi\left(m_{k}\right)+r_{\Omega}\left(m_{k}, m_{k}^{\prime}\right)+r_{\Omega}\left(m_{k}^{\prime}, m\right) \\
& =\varphi\left(m_{k}\right)+r_{\Omega}\left(m_{k}, m\right)=f(m)+\varepsilon_{k} .
\end{aligned}
$$


Переходя к пределу, получим неравенство $\bar{f}^{*}(m) \leqslant \bar{f}(m)$. Это завершает доказательство равенства (11).

Лемма 2. Пусть $\varphi: E \rightarrow \mathbb{R}$, где $E \subset \Omega_{r}-$ некоторое замкнутое множество, $u$

$$
f(m)=\inf _{m^{\prime} \in E}\left\{\varphi\left(m^{\prime}\right)+r_{\Omega}\left(m, m^{\prime}\right)\right\}, \quad m \in \Omega .
$$

Тогда в точках $m \in \Omega \backslash$ д дифберенцируемости функции $f(m)$ выполнено равенство $|\nabla f|=\delta^{-1 / 2}(m)$.

ДокАЗАтЕЛьство. Пусть $m_{0}-$ точка из $\Omega \backslash E$, в которой существует $\nabla f\left(m_{0}\right)$. В силу равенства (11) легко установить, что найдется точка $m_{0}^{\prime} \in \partial D_{\rho}\left(m_{0}\right)$, такая, что

$$
f\left(m_{0}\right)=f\left(m_{0}^{\prime}\right)+r_{\Omega}\left(m_{0}, m_{0}^{\prime}\right)
$$

где $\rho$ выбрано настолько малым, что $D_{\rho}\left(m_{0}\right) \subset \Omega \backslash E$.

Пусть $\gamma(s)$ - геодезическая, соединяющая точки $m_{0}, m_{0}^{\prime}$, и $s$ - ее натуральный параметр. Покажем, что $f(m)=f\left(m_{0}\right)-r_{\Omega}\left(m, m_{0}\right)$ для $m \in \gamma$. Предположим противное. Тогда, так как $\operatorname{Lip}(E, f)=1$, существует точка $m^{\prime} \in \gamma$, такая, что $f\left(m^{\prime}\right)>f\left(m_{0}\right)-r_{\Omega}\left(m^{\prime}, m_{0}\right)$. Имеем

$$
\begin{aligned}
f\left(m_{0}\right)-f\left(m_{0}^{\prime}\right) & =f\left(m_{0}\right)-f\left(m^{\prime}\right)+f\left(m^{\prime}\right)-f\left(m_{0}^{\prime}\right) \leqslant f\left(m_{0}\right)-f\left(m^{\prime}\right)+r_{\Omega}\left(m^{\prime}, m_{0}^{\prime}\right) \\
& <r_{\Omega}\left(m_{0}, m^{\prime}\right)+r_{\Omega}\left(m^{\prime}, m_{0}^{\prime}\right)=r_{\Omega}\left(m_{0}, m_{0}^{\prime}\right) .
\end{aligned}
$$

Мы пришли к противоречию. Следовательно,

$$
\left\langle\nabla f\left(m_{0}\right), \dot{\gamma}(0)\right\rangle=\lim _{s \rightarrow 0} \frac{f(\gamma(s))-f\left(x_{0}\right)}{s}=-\lim _{s \rightarrow 0} \frac{r_{\Omega}\left(\gamma(s), x_{0}\right)}{s}=-\delta^{-1 / 2}\left(m_{0}\right),
$$

и так как $\left|\nabla f\left(m_{0}\right)\right| \leqslant \delta^{-1 / 2}\left(m_{0}\right)$, утверждение доказано.

Следующая теорема составляет основной результат настоящей работы.

Теорема 2. Пусть $M \times{ }_{\delta} \mathbb{R}$ - искривленное лоренцево произведение и $\Omega$ область в нем. Предположим, что множество $\partial \Omega_{r}$ компактно в метрике $r_{\Omega}$ и $\varphi: \partial \Omega_{r} \rightarrow \mathbb{R}$. Тогда для существования функиии $f(m) \in C^{1}(\Omega)$ с изотропным графиком $F$ в $M \times_{\delta} \mathbb{R}$, такой, что $\left.f\right|_{\partial \Omega_{r}}=\varphi$, необходимо и достаточно чтобь выполнялисъ включение $\Omega \subset I_{\varphi}$ и условие (4).

Доказательство. Докажем достаточность. Рассмотрим верхнее и нижнее продолжения $\bar{f}(m)$ и $\underline{f}(m)$ функции $\varphi$. Ясно, что $\bar{f}(m) \geqslant \underline{f}(m)$ при $m \in \Omega_{r}$ и $\bar{f}(m)=\underline{f}(m)=\varphi(m)$ при $m \in \partial \Omega_{r}$. Из теоремы 1 и включения $\Omega \subset I_{\varphi}$ следует тождество $\bar{f} \equiv \underline{f}$.

Докажем, что $\bar{f} \in C^{1}(\Omega)$. Заметим сначала, что из равенства $\bar{f} \equiv \underline{f}$ следует, что для любого геодезического шара $D_{R}\left(m_{0}\right) \subset \Omega$ в силу соотношений $(11)$ и (12) выполняются равенства

$$
\bar{f}(m)=\inf _{m^{\prime} \in \partial D_{R}}\left\{\bar{f}\left(m^{\prime}\right)+r_{\Omega}\left(m^{\prime}, m\right)\right\}, \quad \underline{f}(m)=\inf _{m^{\prime} \in \partial D_{R}}\left\{\underline{f}\left(m^{\prime}\right)+r_{\Omega}\left(m^{\prime}, m\right)\right\} .
$$

Пусть $m_{0} \in \Omega$. Используя лемму 1 , выберем $R>0$ настолько малым, что функция $r_{\Omega}\left(m_{1}, m\right)$ принадлежит $C^{1}\left(D_{R}\left(m_{0}\right)\right)$ по переменной $m$ для любой фиксированной точки $m_{1} \in \partial D_{R}\left(m_{0}\right)$. В силу компактности границы $\partial D_{R}\left(m_{0}\right)$ найдутся точки $m_{1}, m_{2} \in \partial D_{R}\left(m_{0}\right)$, для которых выполняются равенства

$$
\bar{f}\left(m_{0}\right)=\bar{f}\left(m_{1}\right)+r_{\Omega}\left(m_{1}, m_{0}\right), \quad \underline{f}\left(m_{0}\right)=\underline{f}\left(m_{2}\right)-r_{\Omega}\left(m_{2}, m_{0}\right) .
$$


Положим $\bar{v}(m)=\bar{f}\left(m_{1}\right)+r_{\Omega}\left(m_{1}, m\right)$ и $\underline{v}(m)=\underline{f}\left(m_{2}\right)-r_{\Omega}\left(m_{2}, m\right)$. В силу определения функций $\bar{f}, \underline{f}$ для всех $m \in D_{R}\left(m_{0}\right)$ имеем

$$
\underline{v}(m) \leqslant \underline{f}(m)=\bar{f}(m) \leqslant \bar{v}(m) .
$$

Так как $\underline{v}\left(m_{0}\right)=\bar{v}\left(m_{0}\right)$, а функции $\bar{v}, \underline{v}$ принадлежат $C^{1}\left(D_{R}\left(m_{0}\right)\right)$, получаем, что функция $\bar{f}$ дифференцируема в точке $m_{0}$. При этом из леммы 2 следует равенство $\left|\nabla \bar{f}\left(m_{0}\right)\right|=\left|\nabla r_{\Omega}\left(m_{1}, m\right)\right|_{m=m_{0}}=\delta^{-1 / 2}\left(m_{0}\right)$.

Покажем непрерывность $\nabla \bar{f}$ в точке $m_{0}$. Предположим противное. Тогда найдется последовательность $m^{k}$ точек шара $D_{R}\left(m_{0}\right)$, сходящаяся к $m_{0}$, для которой последовательность $\nabla \bar{f}\left(m^{k}\right)$ не сходится к $\nabla \bar{f}\left(m_{0}\right)$. Обозначим через $m_{1}^{k}, m_{2}^{k}$ такие точки границы $\partial D_{R}\left(m_{0}\right)$, что

$$
\bar{f}\left(m^{k}\right)=\bar{f}\left(m_{1}^{k}\right)+r_{\Omega}\left(m_{1}^{k}, m^{k}\right), \quad \bar{f}\left(m^{k}\right)=\underline{f}\left(m^{k}\right)=\underline{f}\left(m_{2}^{k}\right)-r_{\Omega}\left(m_{2}^{k}, m^{k}\right) .
$$

Отметим, что из доказанного выше следует равенство

$$
\nabla \bar{f}\left(m^{k}\right)=\left.\nabla r_{\Omega}\left(m_{1}^{k}, m\right)\right|_{m=m^{k}}
$$

Используя компактность границы $\partial D_{R}\left(m_{0}\right)$, можно выделить подпоследовательности $m_{1}^{k_{l}}$ и $m_{2}^{k_{l}}$, сходящиеся к некоторым точкам $m_{1}^{\prime}, m_{2}^{\prime} \in \partial D_{R}\left(m_{0}\right)$ соответственно. Тогда в силу непрерывности функции $\bar{f}$ получаем

$$
\bar{f}\left(m_{0}\right)=\bar{f}\left(m_{1}^{\prime}\right)+r_{\Omega}\left(m_{1}^{\prime}, m_{0}\right), \quad \bar{f}\left(m_{0}\right)=\bar{f}\left(m_{2}^{\prime}\right)-r_{\Omega}\left(m_{2}^{\prime}, m_{0}\right) .
$$

Таким образом, выполняется равенство $\nabla \bar{f}\left(m_{0}\right)=\left.\nabla r_{\Omega}\left(m_{1}^{\prime}, m\right)\right|_{m=m_{0}}$ и, следовательно,

$$
\nabla \bar{f}\left(m^{k}\right)=\left.\nabla r_{\Omega}\left(m_{1}^{k}, m^{k}\right) \rightarrow \nabla r_{\Omega}\left(m_{1}^{\prime}, m\right)\right|_{m=m_{0}}=\nabla \bar{f}\left(m_{0}\right)
$$

Приходим к противоречию с нашим предположением и, значит, функция $\bar{f}(m)$ является искомым продолжением.

Для доказательства необходимости достаточно заметить, что, во-первых, условие (4) выполняется очевидным образом, а во-вторых, из тождества $\delta^{1 / 2}|\nabla f|$ $\equiv 1$ следует, что для любой точки $m_{0} \in \Omega$ найдутся такие точки $m_{1}, m_{2} \in \partial \Omega_{r}$, в которых выполняются соотношения

$$
f\left(m_{0}\right)=\varphi\left(m_{1}\right)+r_{\Omega}\left(m_{1}, m_{0}\right), \quad f\left(m_{0}\right)=\varphi\left(m_{2}\right)-r_{\Omega}\left(m_{2}, m_{0}\right) .
$$

Для нахождения этих точек необходимо рассмотреть решение $\gamma(s) \Omega$ уравнения $\dot{\gamma}=\nabla f(\gamma)$, для которого $\gamma(0)=m$. Пусть $\left(s_{1}, s_{2}\right)$ - максимальный интервал, на котором определено данное решение. Тогда точки $\gamma\left(s_{1}\right)$ и $\gamma\left(s_{2}\right)$ являются искомыми. Следовательно,

$$
\left|\varphi\left(m_{1}\right)-\varphi\left(m_{2}\right)\right|=r_{\Omega}\left(m_{1}, m_{0}\right)+r_{\Omega}\left(m_{2}, m_{0}\right) \geqslant r_{\Omega}\left(m_{1}, m_{2}\right)
$$

т. е. $m_{0} \in I_{\varphi}$. Теорема доказана.

5. Из доказанных теорем 1 и 2 вытекает следующее утверждение.

Теорема 3. Пусть $M \times_{\delta} \mathbb{R}-$ искривленное лоренцево произведение, где многообразие $M$ и функиия $\delta$ принадлежат классу $C^{2}$. Пусть $\Omega \subset M-$ подоб- 
ласть с компактной в метрике (2) границей $\partial \Omega_{r}$, и пусть $\varphi: \partial \Omega_{r} \rightarrow \mathbb{R}-$ липшищева в метрике $r_{\Omega}$ функиия. Предположим, что для множества единственности функиии ч выполняется равенство $U_{\varphi}=\Omega$. Пусть $\tilde{f}=\left.\underline{f}\right|_{\Omega}=\left.\bar{f}\right|_{\Omega}$. Тогда $\tilde{f} \in C^{1}(\Omega)$ и величина $\delta^{1 / 2}(m)|\nabla \tilde{f}(m)|$ постоянна в $\Omega$, причем

$$
\delta^{1 / 2}(m)|\nabla \tilde{f}(m)| \equiv \sup _{\substack{m^{\prime}, m^{\prime \prime} \in \partial \Omega_{r} \\ m^{\prime} \neq m^{\prime \prime}}} \frac{\left|\varphi\left(m^{\prime}\right)-\varphi\left(m^{\prime \prime}\right)\right|}{r_{\Omega}\left(m^{\prime}, m^{\prime \prime}\right)} .
$$

ДокАЗАТЕЛьство. Как и при доказательстве предыдущей теоремы, можно считать, что

$$
\sup _{\substack{m^{\prime}, m^{\prime \prime} \in \partial \Omega_{r} \\ m^{\prime} \neq m^{\prime \prime}}} \frac{\left|\varphi\left(m^{\prime}\right)-\varphi\left(m^{\prime \prime}\right)\right|}{r_{\Omega}\left(m^{\prime}, m^{\prime \prime}\right)}=1 .
$$

Тогда, согласно теореме $1, I_{\varphi}=U_{\varphi}=\Omega$, и из теоремы 2 следует, что $\tilde{f}=\bar{f} \in$ $C^{1}(\Omega)$, причем $\delta^{1 / 2}(m)|\nabla \tilde{f}(m)| \equiv 1$.

Для графиков в $\mathbb{R}^{n}$ соответствующее утверждение установлено Аронсоном $[6]$.

6. Заметим, что в силу изотропности поверхность $F$ «состоит» из отрезков световых прямых, т. е. прямых, в каждой точке которых касательный вектор является световым. Однако изотропного продолжения может и не существовать, если условие $\Omega \subset I_{\varphi}$ заменить более слабым: любую точку $\left(m_{1}, \varphi\left(m_{1}\right)\right)$ контура можно соединить с некоторой точкой $\left(m_{2}, \varphi\left(m_{2}\right)\right)$ контура световой линией. Действительно, рассмотрим следующий пример.

Пусть $M=\mathbb{R}^{2}$ и $\delta \equiv 1$, т. е. $M \times{ }_{\delta} \mathbb{R}$ есть пространство-время Минковского $\mathbb{R}_{1}^{3}$. В качестве области $\Omega$ рассмотрим шар $B=\left\{\left(x_{1}, x_{2}\right): x_{1}^{2}+x_{2}^{2}<1\right\}$. Разобьем окружность $\partial B$ на четыре дуги точками $(1 / \sqrt{2}, 1 / \sqrt{2}),(1 / \sqrt{2},-1 / \sqrt{2})$, $(-1 / \sqrt{2},-1 / \sqrt{2}),(-1 / \sqrt{2}, 1 / \sqrt{2})$. Обозначим их, начиная с верхней и по часовой стрелке, через $S_{1}, S_{2}, S_{3}, S_{4}$ соответственно. Тогда функция

$$
\varphi(x)= \begin{cases}x_{1} & \text { при } x \in S_{1}, \\ x_{2} & \text { при } x \in S_{2}, \\ -x_{1} & \text { при } x \in S_{3}, \\ -x_{2} & \text { при } x \in S_{4}\end{cases}
$$

обладает перечисленными выше свойствами. При этом $I_{\varphi}=\bar{B} \backslash K$, где $K-$ открытый квадрат $(-1 / \sqrt{2}, 1 / \sqrt{2}) \times(-1 / \sqrt{2}, 1 / \sqrt{2})$, т. е. $B$ не содержится в $I_{\varphi}$. Из доказанной теоремы следует, что не существует изотропного гладкого продолжения функции $\varphi$ во всю область $B$.

Отметим также существенность условия (4). Достаточно рассмотреть следующий пример. Пусть $\Omega=\left\{x=\left(x_{1}, x_{2}\right): 1<x_{1}^{2}+x_{2}^{2}<R^{2}\right\}$ и функция $\varphi$ равна 0 при $|x|=1$ и $\sqrt{R^{2}-1}$ при $|x|=R$. Ясно, что изотропного продолжения не существует, несмотря на то, что множество $I_{\varphi}$ содержит область $\Omega$.

Авторы признательны Владимиру Александровичу Клячину, прочитавшему рукопись настоящей статьи и сделавшему ряд ценных замечаний по тексту. Авторы также выражают благодарность Виктору Владимировичу Леонтьеву за помощь в работе с текстом статьи. 


\section{ЛитерАТУРА}

1. Бим Дж., Эрлих П. Глобальная лоренцева геометрия. Мир, М., 1990.

2. Клячин A. A., Миклюков B. M. Следы функций с пространственноподобными графиками и задача о продолжении при ограничениях на градиент. Матем. сб., 183, №7, 49-64 (1992).

3. Grigoryeva E., Klyachin A., Miklyukov V. Problem of functional extension and spacelike surfaces in Minkowski space. Z. Anal. Anwendungen, 21, 719-752 (2002).

4. Bartnik R., Simon L. Spacelike hypersurfaces with prescribed boundary values and mean curvature. Commun. Math. Phys., 87, 131-152 (1982/83).

5. Клячин A. A., Миклюков B. М. Существование решений с особенностями уравнения максимальных поверхностей в пространстве Минковского. Матем. сб., 184, №9, 103-124 (1993).

6. Aronsson G. Extension of functions satisfying Lipschitz conditions. Ark. Mat., 6, No. 28, 551-561 (1967).

7. Jensen $R$. Uniqueness of Lipschitz extension: minimizing the sup norm of the gradient. Arch. Rational Mech. Anal., 123, 51-74 (1993).

8. Crandall M. G., Evans L. C., Gariepy R. F. Optimal Lipschitz extensions and the infinity Laplacian. Calc. Var. Partial Differential Equations, 13, No. 2, 123-139 (2001).

9. Juutinen $P$. Absolutely minimizing Lipschitz extensions on a metric space. Ann. Acad. Sci. Fenn., Ser. AI Math., 27, 57-67 (2002).

10. Федерер Г. Геометрическая теория меры. Наука, М., 1987.

11. Дубровин Б. А., Новиков С. П., Фоменко А. Т. Современная геометрия. Наука, M., 1979.

12. Бишоп Р. Л., Криттенден Р. Дж. Геометрия многообразий. Мир, М., 1967.

13. McShane E. J. Extension of range of functions. Bull. Amer. Math. Soc., 40, 837-842 (1934).

14. Whitney $H$. Analytic extensions of differentiable functions defined in closed sets. Trans. Amer. Math. Soc., 36, 63-89 (1934).

15. Кобаяси Ш., Номидзу К. Основы дифференциальной геометрии. Т. 2. Наука, М., 1981.

Волгоградский государственный университет, математический факультет

Поступило в редакцию e-mail: klchna@zmail.ru 13 октября 2003 г.

Волгоградский государственный университет, математический факультет

e-mail:miklyuk@mail.ru 